\title{
A Mathematical Model of Three-Dimensional Flow in a Scraped-Surface Heat Exchanger
}

\author{
Stephen K. Wilson ${ }^{1}$, Brian R. Duffy ${ }^{1}$ and Michael E. M. Lee ${ }^{2}$ \\ 1 Department of Mathematics, University of Strathclyde, 26 Richmond Street, \\ Glasgow, G1 1XH, UK s.k.wilson@strath.ac.uk, b.r.duffy@strath.ac.uk \\ 2 School of Mathematics, University of Southampton, Highfield, Southampton, \\ SO17 1BJ, UK m.e.m.lee@soton.ac.uk
}

Summary. We present a simple mathematical model of fluid flow in a ScrapedSurface Heat Exchanger (SSHE). Specifically we consider steady isothermal flow of a Newtonian fluid around a periodic array of pivoted scraper blades in a channel with one stationary and one moving wall, when there is an applied pressure gradient in a direction perpendicular to the wall motion. The flow is fully three-dimensional, but decomposes naturally into a two-dimensional transverse flow driven by the boundary motion and a longitudinal pressure-driven flow.

Key words: Mathematical Modelling, Scraped-Surface Heat Exchanger

\section{Scraped-Surface Heat Exchangers (SSHEs)}

Scraped-surface heat exchangers (SSHEs) are widely used in the food industry to cook, chill or sterilize certain foodstuffs quickly and efficiently without causing unwanted changes to the constitution, texture and appearance of the final product. A SSHE is essentially a cylindrical steel annulus whose outer wall is heated or cooled externally; the foodstuff is driven slowly by an axial pressure gradient along the annulus, and a "bank" of blades rotating with the inner wall (the "rotor") is used to scrape it away from the outer wall (the "stator"), preventing fouling, and maintaining mixing and heat transfer. The blades typically are arranged in groups of two $\left(180^{\circ}\right.$ apart $)$ or four $\left(90^{\circ}\right.$ apart $)$; sometimes pairs of blades are "staggered" axially. The processes that take place inside SSHEs are complex; operating conditions vary with context, and operators are guided largely by experience and empirical correlations. Typically SSHEs are used on highly viscous foodstuffs; examples include purées, sauces, margarines, jams, spreads, soups, baby-foods, chocolate, mayonnaise, caramel, fudge, ice-cream, cream and yoghurt. Such foodstuffs commonly behave as non-Newtonian materials, typically being shear-thinning, viscoplastic and/or viscoelastic, as well as being inhomogeneous, and possibly undergoing 


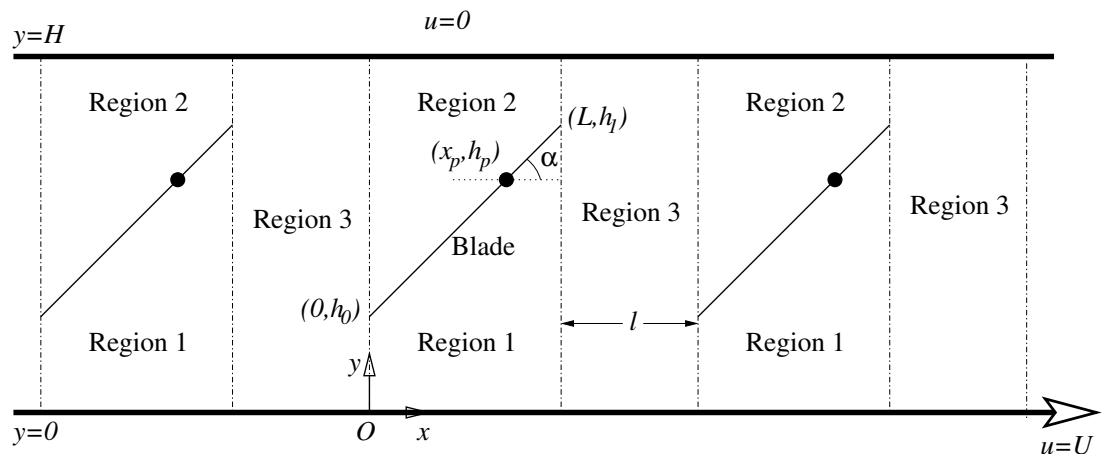

Fig. 1. Geometry of the transverse flow problem.

phase changes; also they often have a strongly temperature-dependent viscosity. Moreover, both convection and dissipation of heat can be significant in a SSHE. Extensive literature surveys are given by Härröd [2, 3], and features of the behaviour have recently been analysed by Stranzinger et al. [4], Fitt and Please [1] and Sun et al. [5]. However, despite their widespread use, understanding of the behaviour of the material inside SSHEs is still incomplete.

In the present work we shall concentrate on the fluid flow rather than the heat transfer inside a SSHE, and so we shall restrict our attention to isothermal flow.

\section{Transverse Flow}

First we consider steady two-dimensional flow of an isothermal incompressible Newtonian fluid of viscosity $\mu$ in a long parallel-sided channel of width $H$ in which there is a periodic array of inclined smoothly pivoted thin plane blades, the flow being driven by the motion of one wall of the channel parallel to itself with speed $U(>0)$, the other wall being fixed. Body forces are neglected.

We introduce Cartesian axes $O x y z$ as shown in Figure 1, with the wall $y=0$ moving with velocity $U \mathbf{i}$, and the wall $y=H$ fixed. Suppose a thin plane freely pivoted blade occupies $0 \leq x \leq L$, with its pivot fixed at $\left(x_{\mathrm{p}}, h_{\mathrm{p}}\right)$, where $0 \leq x_{\mathrm{p}} \leq L$ and $0<h_{\mathrm{p}}<H \ll L$, and suppose that the separation between the blades is $\ell(\geq 0)$, so that the portion $L \leq x \leq L+\ell$ of the channel contains no blades. Let $\alpha$ (which may be positive or negative) denote the angle of inclination of the blade to the $x$ axis, as shown in Figure 1. In the lubrication-theory approach used here we will assume that $|\alpha| \ll 1$; then the blade is given by $y=h(x)$ for $0 \leq x \leq L$, where $h(x)=h_{\mathrm{p}}+\alpha\left(x-x_{\mathrm{p}}\right)$.

For steady flow the blade is in equilibrium, subject to forces due to the fluid, the pivot, and (in general) the walls of the channel. Here we consider cases where the ends of the blade are not in contact with the moving wall $y=0$ of the channel, so that $0<h_{0}, h_{1} \leq H$, where $h_{0}=h(0)$ and $h_{1}=h(L)$. 
We denote the velocities, pressures and volume fluxes (per unit width in the $z$ direction) by $u_{k} \mathbf{i}+v_{k} \mathbf{j}, p_{k}$ and $Q_{k}$, where $k=1$ denotes values in $0 \leq x \leq L, 0 \leq y \leq h, k=2$ denotes values in $0 \leq x \leq L, h \leq y \leq H$, and $k=3$ denotes values in $L \leq x \leq L+\ell, 0 \leq y \leq H$. A lubrication approximation gives

$$
\begin{gathered}
u_{1}=\frac{\left[6 Q_{1} y+U h(h-3 y)\right](h-y)}{h^{3}}, \\
u_{2}=\frac{6 Q_{2}(H-y)(y-h)}{(H-h)^{3}}, \\
u_{3}=\frac{\left[6 Q_{3} y+U H(H-3 y)\right](H-y)}{H^{3}} . \\
p_{1}=\frac{6 \mu U}{\alpha}\left(\frac{1}{h_{1}}-\frac{1}{h}\right)-\frac{6 \mu Q_{1}}{\alpha}\left(\frac{1}{h_{1}^{2}}-\frac{1}{h^{2}}\right)+p_{L}, \\
p_{2}=\frac{6 \mu Q_{2}}{\alpha}\left[\frac{1}{\left(H-h_{1}\right)^{2}}-\frac{1}{(H-h)^{2}}\right]+p_{L}, \\
p_{3}=\frac{6 \mu\left(U H-2 Q_{3}\right)}{H^{3}}(x-L)+p_{L}, \\
Q_{1}=\int_{0}^{h} u_{1} \mathrm{~d} y=-\frac{h^{3} p_{1 x}}{12 \mu}+\frac{U h}{2}, \\
Q_{2}=\int_{h}^{H} u_{2} \mathrm{~d} y=-\frac{(H-h)^{3} p_{2 x}}{12 \mu}, \\
Q_{3}=\int_{0}^{H} u_{3} \mathrm{~d} y=-\frac{H^{3} p_{3 x}}{12 \mu}+\frac{U H}{2} .
\end{gathered}
$$

Setting $x=0$ in (4) and (5) and $x=L+\ell$ in (6), we obtain three representations of $p_{0}-p_{L}$ :

$$
\begin{array}{r}
p_{0}-p_{L}=-\frac{6 \mu U}{\alpha}\left(\frac{1}{h_{0}}-\frac{1}{h_{1}}\right)+\frac{6 \mu Q_{1}}{\alpha}\left(\frac{1}{h_{0}^{2}}-\frac{1}{h_{1}^{2}}\right) \\
=-\frac{6 \mu Q_{2}}{\alpha}\left[\frac{1}{\left(H-h_{0}\right)^{2}}-\frac{1}{\left(H-h_{1}\right)^{2}}\right]=\frac{6 \mu\left(U H-2 Q_{3}\right) \ell}{H^{3}} .
\end{array}
$$

Expressions for the $Q_{k}(k=1,2,3)$ and $p_{0}-p_{L}$ are obtained by solving (10) and the global mass conservation condition $Q_{1}+Q_{2}=Q_{3}$.

The moment of the forces on the blade about the pivot due to the pressure is of the form $\mathbf{M}=M \mathbf{k}$, where

$$
M=\int_{0}^{L}\left(x-x_{\mathrm{p}}\right)\left(p_{1}-p_{2}\right) \mathrm{d} x .
$$

For equilibrium of the blade we require $M=0$, which leads to a lengthy algebraic transcendental equation determining $\alpha$ when $L, \ell, H, x_{\mathrm{p}}$ and $h_{\mathrm{p}}$ 
are prescribed. Once $\alpha$ is known, the complete solution is determined. This solution allows us to describe all the qualitative features of the transverse flow. In particular, we can determine when the blades are in contact with the walls of the channel. In addition we can calculate the forces on the blades and on the walls of the channel, and hence make useful estimates of the torque and power required to turn the rotor.

\section{Longitudinal Flow}

In a SSHE the material being processed not only undergoes flow in the transverse direction (caused by the rotation of the rotor), but also is driven by an imposed axial pressure gradient along the annular gap between stator and rotor. To model this fully three-dimensional flow we consider the effect of allowing flow along the channel in the $z$ direction, in addition to the flow in the $(x, y)$ plane, discussed above. We take the blades to be long in the $z$ direction so that the lubrication approximation may again be used. It is found that the motion in the $z$ direction uncouples from that in the $(x, y)$ plane. Thus with velocities and pressures denoted by $u_{k} \mathbf{i}+v_{k} \mathbf{j}+w_{k} \mathbf{k}$ and $P_{k}$ for $k=1,2,3$ (with $u_{k}, v_{k}, w_{k}$ and $P_{k}$ functions of $x, y$ and $z$ ), we find that

$$
w_{1}=\frac{G}{2 \mu} y(h-y), \quad w_{2}=\frac{G}{2 \mu}(H-y)(y-h), \quad w_{3}=\frac{G}{2 \mu} y(H-y),
$$

and $P_{k}=-G z+p_{k}$, where $G=-\partial P_{k} / \partial z$ is the (constant) prescribed axial pressure gradient, and the velocity components $u_{k}=u_{k}(x, y)$ and $v_{k}=v_{k}(x, y)$ and the pressure contributions $p_{k}=p_{k}(x, y)$ are exactly as given for the transverse (two-dimensional) flow described above. The volume flux of fluid in the $z$ direction across the section $0 \leq x \leq L+\ell, 0 \leq y \leq H$ is given by

$$
Q_{z}=\frac{G H}{24 \mu}\left[L\left(2\left(h_{0}^{2}+h_{0} h_{1}+h_{1}^{2}\right)-3 H\left(h_{0}+h_{1}\right)\right)+2 H^{2}(L+\ell)\right] .
$$

The force (per unit axial length) in the $z$ direction on the blade due to the fluid is given by $F_{z}=G H L / 2$, and the forces (per unit axial length) in the $z$ direction on the portions $0 \leq x \leq L+\ell$ of the lower wall $y=0$ and the upper wall $y=H$ due to the fluid are

$$
F_{0}=\left.\int_{0}^{L} \mu \frac{\partial w_{1}}{\partial y}\right|_{y=0} \mathrm{~d} x+\left.\int_{L}^{L+\ell} \mu \frac{\partial w_{3}}{\partial y}\right|_{y=0} \mathrm{~d} x=\frac{G}{4}\left[2 H \ell+\left(h_{0}+h_{1}\right) L\right]
$$

and

$F_{H}=-\left.\int_{0}^{L} \mu \frac{\partial w_{2}}{\partial y}\right|_{y=H} \mathrm{~d} x-\left.\int_{L}^{L+\ell} \mu \frac{\partial w_{3}}{\partial y}\right|_{y=H} \mathrm{~d} x=\frac{G}{4}\left[2 H(L+\ell)-\left(h_{0}+h_{1}\right) L\right]$,

respectively. 


\section{Summary}

In this short paper we presented a simple mathematical model of fluid flow in a SSHE. Specifically we considered steady isothermal flow of a Newtonian fluid around a periodic array of pivoted scraper blades in a channel with one stationary and one moving wall, when there is an applied pressure gradient in a direction perpendicular to the wall motion. The flow is fully three-dimensional, but decomposes naturally into a two-dimensional transverse flow driven by the boundary motion and a longitudinal pressure-driven flow. In the future we plan to extend our analysis to include other practically important features neglected in this simple model, including blade wear and, of course, nonisothermal effects.

\section{Acknowledgements}

Thanks are due to Profs C. P. Please and A. D. Fitt, School of Mathematics, University of Southampton, Prof. D. L. Pyle and Dr K.-H. Sun, School of Food Biosciences, University of Reading, Dr N. Hall Taylor, Chemtech International Ltd, Reading, Dr J. Mathisson, Tetra Pak, and Dr H. Tewkesbury, Smith Institute for Industrial Mathematics and System Engineering, for many useful discussions. This work forms part of a larger research project supported by the EPSRC (Research Grant GR/R993032), and by Chemtech International and Tetra Pak, under the auspices of the Faraday Partnership for Industrial Mathematics, managed by the Smith Institute.

\section{References}

1. A. D. Fitt and C. P. Please. Asymptotic analysis of the flow of shear-thinning foodstuffs in annular scraped heat exchangers. J. Eng. Maths, 39:345-366, 2001.

2. M. Härröd. Scraped surface heat exchangers: a literature survey of flow patterns, mixing effects, residence time distribution, heat transfer and power requirements. J. Food Proc. Eng., 9:1-62, 1986.

3. M. Härröd. Methods to distinguish between laminar and vortical flow in scraped surface heat exchangers. J. Food Proc. Eng., 13:39-57, 1990.

4. M. Stranzinger, K. Feigl, and E. Windhab. Non-Newtonian flow behaviour in narrow annular gap reactors. Chem. Eng. Sci., 56:3347-3363, 2001.

5. K.-H. Sun, D. L. Pyle, A. D. Fitt, C. P. Please, M. Baines, and N. Hall-Taylor. Numerical study of $2 \mathrm{D}$ heat transfer in a scraped surface heat exchanger. Computers and Fluids, 33:869-880, 2003. 